\title{
Clostridium celatum
}

National Cancer Institute

\section{Source}

National Cancer Institute. Clostridium celatum. NCI Thesaurus. Code C86275.

A species of anaerobic, Gram positive, rod shaped bacteria assigned to the phylum

Firmicutes. This species is non-motile, sporeforming, urease positive, catalase and indole negative and reduces sulfites and nitrates. C. celatum is part of the normal flora of the gastrointestinal tract and has been isolated from clinically normal human feces. 